\title{
Propagating waves in polar coronal holes as seen by SUMER \& EIS ${ }^{\star}$
}

\author{
D. Banerjee ${ }^{1}$, L. Teriaca ${ }^{2}$, G. R. Gupta ${ }^{1,3}$, S. Imada ${ }^{4}$, G. Stenborg ${ }^{5}$, and S. K. Solanki ${ }^{2,6}$ \\ 1 Indian Institute of Astrophysics, Bangalore 560034, India \\ e-mail: dipu@iiap.res.in \\ 2 Max-Planck-Institut für Sonnensystemforschung (MPS), 37191 Katlenburg-Lindau, Germany \\ 3 Joint Astronomy Programme, Indian Institute of Science, Bangalore 560012, India \\ 4 National Astronomical Observatory of Japan, 2-21-1 Osawa, Mitaka, Tokyo 181-8588, Japan \\ 5 Interferometrics, Inc., Herndon, VA 20171, USA \\ 6 School of Space Research, Kyung Hee University, Yongin, Gyeonggi 446-701, Korea
}

Received 13 March 2009 / Accepted 22 April 2009

ABSTRACT

\begin{abstract}
Context. To study the dynamics of coronal holes and the role of waves in the acceleration of the solar wind, spectral observations were performed over polar coronal hole regions with the SUMER spectrometer on SoHO and the EIS spectrometer on Hinode. Aims. Using these observations, we aim to detect the presence of propagating waves in the corona and to study their properties. Methods. The observations analysed here consist of SUMER spectra of the Ne VIII $770 \AA$ Aine $(T=0.6 \mathrm{MK})$ and EIS slot images in the Fe XII $195 \AA ̊$ line $(T=1.3 \mathrm{MK})$. Using the wavelet technique, we study line radiance oscillations at different heights from the limb in the polar coronal hole regions.

Results. We detect the presence of long period oscillations with periods of 10 to $30 \mathrm{~min}$ in polar coronal holes. The oscillations have an amplitude of a few percent in radiance and are not detectable in line-of-sight velocity. From the time distance maps we find evidence for propagating velocities from $75 \mathrm{~km} \mathrm{~s}^{-1}$ (Ne VIII) to $125 \mathrm{~km} \mathrm{~s}^{-1}$ (Fe XII). These velocities are subsonic and roughly in the same ratio as the respective sound speeds.

Conclusions. We interpret the observed propagating oscillations in terms of slow magneto-acoustic waves. These waves can be important for the acceleration of the fast solar wind.
\end{abstract}

Key words. Sun: corona - Sun: oscillations - Sun: UV radiation - Sun: transition region - waves

\section{Introduction}

Propagating radiance oscillations were detected in polar plumes, first by Ofman et al. (1997) using UVCS/SoHO and later by Deforest \& Gurman (1998) with EIT/SoHO. Ofman et al. (1999, 2000a) identified the observed radiance oscillations as propagating slow magneto-acoustic waves. A number of studies using the $\mathrm{CDS} / \mathrm{SoHO}$ spectrometer have reported oscillations in the polar coronal holes up to $25^{\prime \prime}$ above the limb (e.g., Banerjee et al. 2000, 2001a,b; O'Shea et al. 2006, 2007). Popescu et al. (2005), using SUMER/SoHO, detected radiance fluctuations with periods from 10 to $90 \mathrm{~min}$ up to $15^{\prime \prime}$ above the limb. These studies point to the presence of compressional waves, thought to be slow magneto-acoustic waves. In this letter, for the first time to our knowledge, simultaneous use of the SUMER and EIS/Hinode spectrometer were used to study these propagating disturbances in the off-limb regions of the polar coronal holes. We construct time distance maps to study the properties of wave propagation and use wavelet analysis to establish their periods. Spectroscopic observations have the advantage of a narrow temperature response (by isolating specific spectral lines) and of allowing the study of resolved and unresolved plasma motions by measuring the Doppler shift and width of the observed profiles. These observables provide important constraints in establishing the nature of the observed oscillations.

\footnotetext{
* Figures 6 and 7 are only available in electronic form at http://www . aanda.org
}

\section{Observations}

The data analysed here were obtained on 8th and 15th April 2007 during a Hinode/SUMER joint observing campaign as part of the Hinode Observing Programme (HOP) 45/Joint Observing program (JOP) 196. They consist of time series taken in the south polar coronal hole by the Solar Ultraviolet Measurements Of Emitted Radiation (SUMER, Wilhelm et al. 1995) spectrometer aboard the Solar and Heliospheric Observatory (SoHO) and by the EUV imaging spectrometer (EIS, Culhane et al. 2007) aboard Hinode (Kosugi et al. 2007). For SUMER, the $1^{\prime \prime} \times 120^{\prime \prime}$ slit was centred on the limb and spectra were acquired from 19:13 to $20: 47$ UTC on 8th and from 10:44 to 14:45 UTC on 15 th, with a cadence of $18.12 \mathrm{~s}$. For EIS, the $40^{\prime \prime}$ wide slot was used to obtain $40^{\prime \prime} \times 160^{\prime \prime}$ images in several spectral lines over the time interval $18: 42$ to $20: 58$ UTC on 8 th and 10:54 to 15:57 UTC on 15th April. The EIS data consist of a series of elementary rasters each formed by two slot images displaced by $20^{\prime \prime}$ in the $X$ direction to maximise the chances of overlapping with the other instruments. Each slot image was exposed for about $7.5 \mathrm{~s}$. As a result, for each dataset, we have two time series with a cadence of $19.3 \mathrm{~s}$ that we identify hereafter with slot $_{0}$ (East) and slot 1 (West). Before the start of the temporal series, raster images were obtained with SUMER and EIS to allow the co-alignment of the different instruments. 

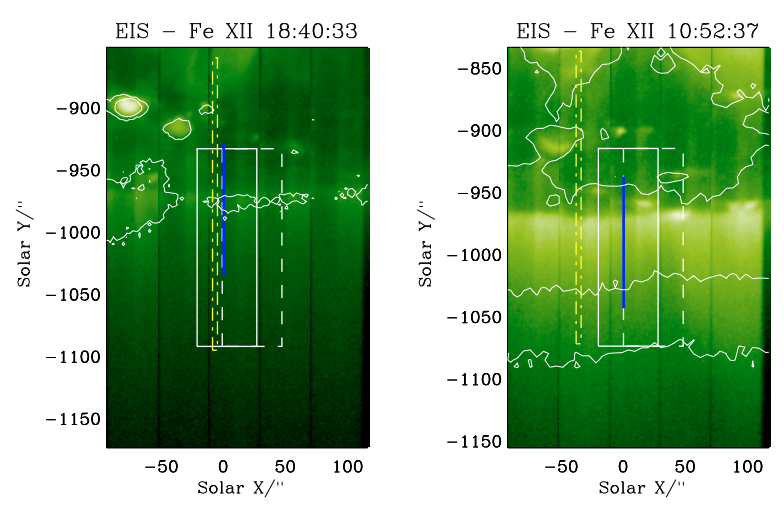

Fig. 1. Left (right) panel shows the location of different slits and slots on the Fe XII $195 \AA$ EIS context raster on 8th April (15th April). The context rasters are made of five adjacent slot images, covering a $200^{\prime \prime} \times 320^{\prime \prime}$ area. In both panels, radiance iso-contours (white) are from EIT Fe XII $195 \AA$ images. The two white rectangular boxes are the location of the EIS slots (solid slot 0 , dashed slot ${ }_{1}$ ). The solid vertical blue line represents the location of the SUMER $1^{\prime \prime}$ wide slit whereas the yellow dash-dot elongated box gives the location of the 4 " wide CDS slit.

All the data were reduced and calibrated with the standard procedures given in the SolarSoft $(\mathrm{SSW})^{1}$ library. SUMER data were decompressed, corrected for response inhomogeneities (flat-field) and for geometrical distortion (de-stretch). The data series of 8th April was analysed only until 20:47 UTC due to spurious flaring at the edges of the detector occurring after this time. For EIS, after applying the standard reduction and calibration provided by the eis_prep procedure, data were corrected for the spacecraft jitter in the $Y$-direction (the jitter in the $X$ direction is less than $1^{\prime \prime}$ and can be neglected) by using housekeeping data. Finally, the movement of the slot image on the detector due to thermal variations during the orbit was corrected. The displacement in the dispersion (Solar $X$ ) direction was obtained by measuring the position of the edge of the Fe XII 195 slot image over time. The displacement in the $Y$ direction is taken equal to 1.5 times that in the $X$ direction (Imada 2009, in preparation). The validity of the latter assumption was verified by checking the limb Y position vs. time. All images from Hinode were converted to SoHO view (L1) and co-aligned in two steps. First, the internal offset between the long-wavelength and shortwavelength EIS CCDs and the wavelength dependent inclination of the spectrum were obtained (Kamio \& Hara, private communication) and accounted for. Then, choosing SUMER as the reference, the EIS rasters were aligned with the SUMER rasters. We estimate the alignment to be accurate within $2^{\prime \prime}$. Figure 1 shows the location of the slits and slots of the different instruments. Here the white rectangular boxes represent EIS slot ${ }_{0}$ and slot $_{1}$. The vertical lines indicates the location of the SUMER (solid blue) and CDS (dash-dotted yellow) slits. Due to the marginal overlap with the other instruments and the low signal in the off-limb spectra, CDS data were not used for the analysis. SUMER data overlap with EIS slot ${ }_{0}$ in both cases and only those data are used here.

\section{Results}

Maps of the radiance along the slit vs. time (xt slices) were first built using the SUMER Ne VIII integrated line radiances and

${ }^{1}$ http://sohowww . nascom.nasa.gov/solarsoft/
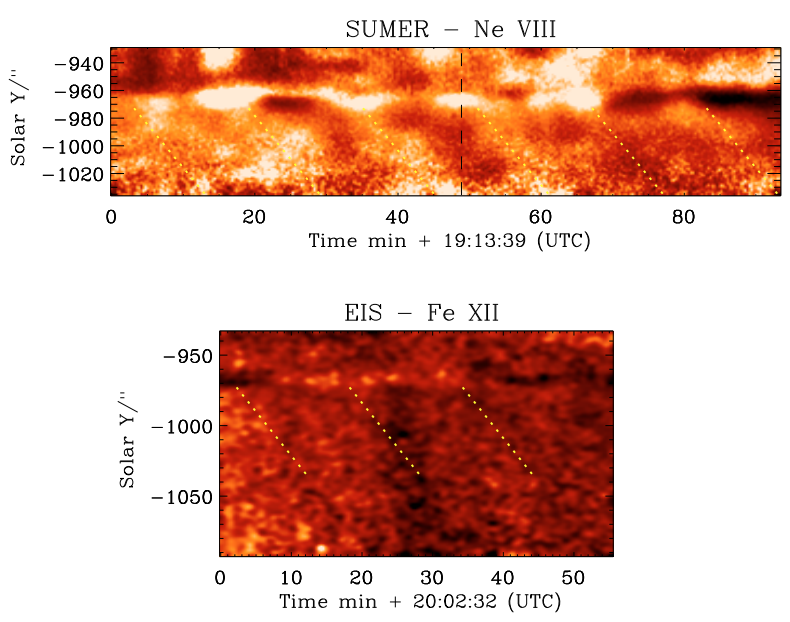

Fig. 2. Enhanced (see text) maps of radiance variation along the slit (Solar $Y$ direction) with time for Ne VIII as recorded by SUMER (top panel) and Fe XII $195 \AA$ as recorded by EIS (bottom panel) on 8th April 2007. The vertical black dashed line on the SUMER enhanced radiance map depicts the starting point of the EIS time series (shown in the bottom panel). The slanted dotted yellow lines correspond to disturbances propagating with a speed of $75 \mathrm{~km} \mathrm{~s}^{-1}$ and a period of about $15 \mathrm{~min}$, as determined from the SUMER data.
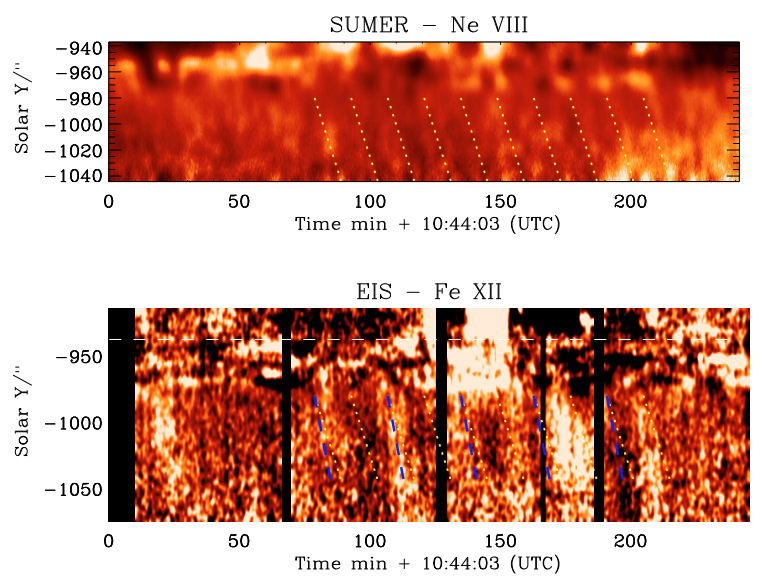

Fig. 3. Enhanced radiance maps as in Fig. 2, but for the 15th April data. Black vertical stripes correspond to data gaps in the EIS time series. The horizontal white dashed line on the EIS enhanced radiance map depicts the upper edge of the SUMER FOV. The slanted dotted yellow lines correspond to disturbances propagating with a speed of $75 \mathrm{~km} \mathrm{~s}^{-1}$ and a period of about $15 \mathrm{~min}$ as determined from the SUMER data. The slanted dashed blue lines on the EIS map correspond to disturbances propagating with a speed of $\sim 125 \mathrm{~km} \mathrm{~s}^{-1}$ and a period of about $30 \mathrm{~min}$ as determined from the EIS data.

EIS Fe XII radiances averaged over $5^{\prime \prime}$ in the $X$ direction at the position overlapping the SUMER slit. The resulting maps were then contrast enhanced and low-pass filtered to produce the maps shown in Figs. 2 and 3 for the 8th and 15th April datasets, respectively. EIS radiance maps were normalised by dividing by the average along the $Y$ direction (to remove the strong limb signature), the result elevated to the power of 3 (to increase the contrast between high/low radiance regions), and, finally, lowpass filtered. SUMER enhanced radiance maps were obtained as the median over $3 \times 3$ pixels of the quantity $\exp (a+0.15 \times b)^{2}$ where $b$ is the original image, and a is a highly smoothed version of the original image obtained by convolving with an isotropic 


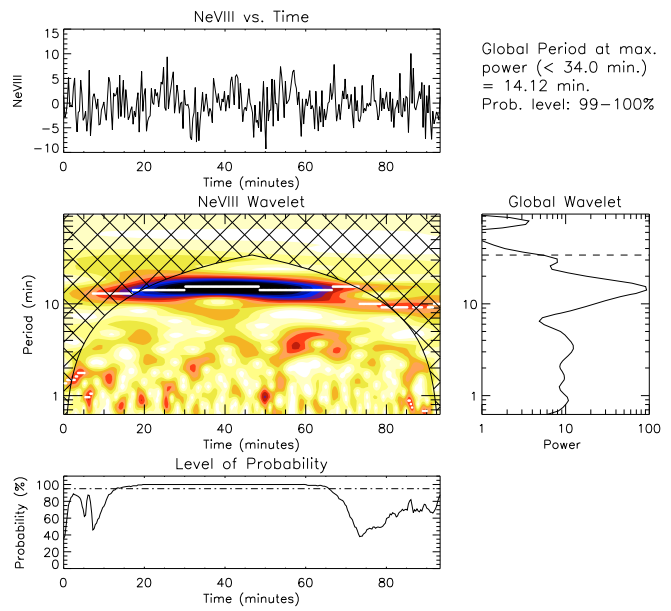

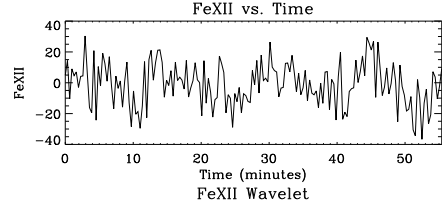

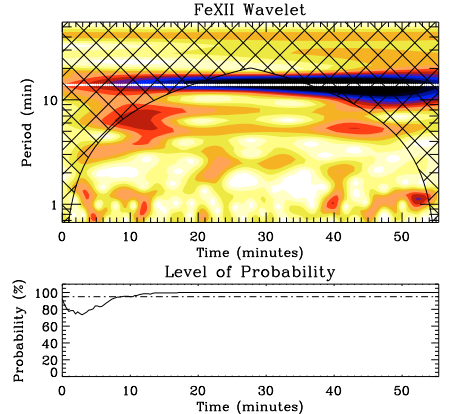

Global Period at max power $=13.89 \mathrm{~min}$ $=13.89$ min.
Prob. level: $99-100 \%$

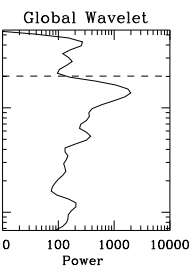

Fig. 4. Wavelet analysis for the 8th April data at Solar $Y \sim-995^{\prime \prime}$ for both Ne VIII $770 \AA$ (left) and Fe XII $195 \AA$ (right). Both light curves are obtained by averaging over $9^{\prime \prime}$ along the slit. In each set we show the relative (background trend-removed) radiance (top panels), the colour inverted wavelet power spectrum (central panels), the variation of the probability estimate associated with the maximum power in the wavelet power spectrum (marked with white lines) (bottom panels), and the global (averaged over time) wavelet power spectrum (right middle panels). Above the global wavelet, the period (measured from the maximum power of the global wavelet), and the probability estimate, are given.
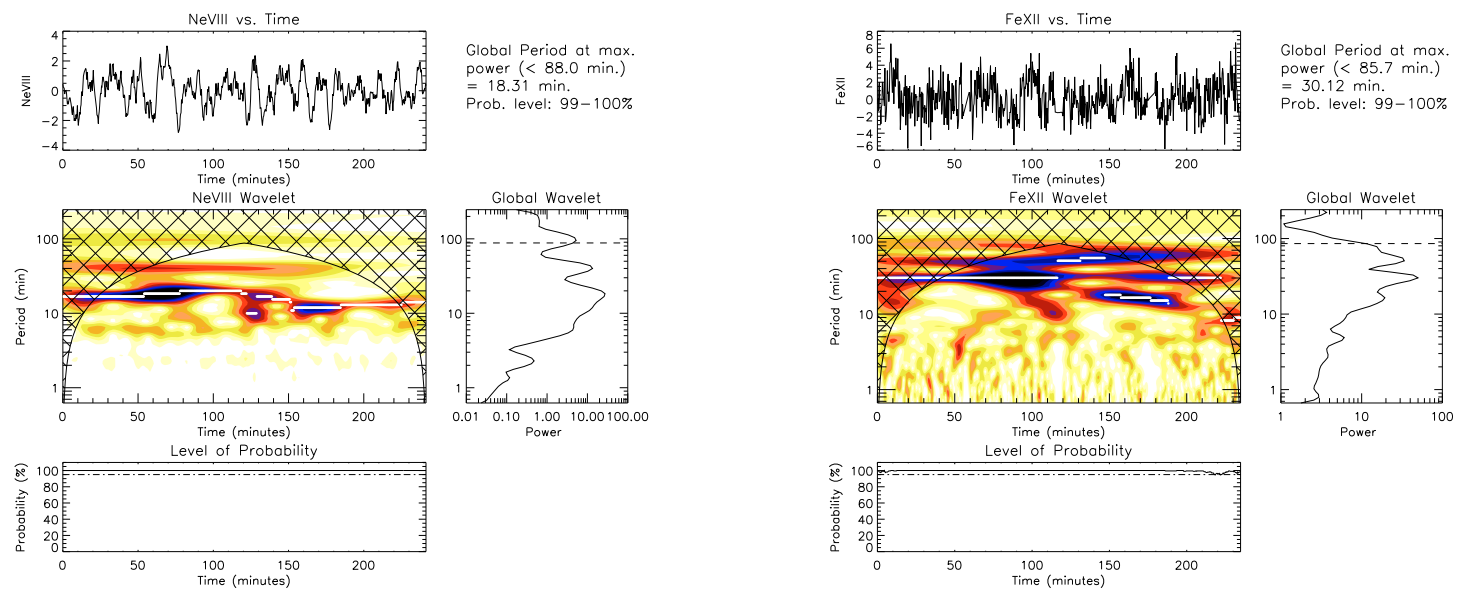

Fig. 5. Wavelet analysis for the 15th April data at Solar $Y \sim-995^{\prime \prime}$ for both Ne VIII $770 \AA$ A (left) and Fe XII $195 \AA$ A (right). Both light curves are obtained by averaging over $9 "$ along the slit. Panels as in Fig. 4.

kernel. The difference in the treatment of the data from the two instruments depends on the different noise level and image scale. In Fig. 2, we note that the EIS time series starts at a later time (denoted by a dashed vertical line in the top panel of the figure). On xt slices, the presence of alternate bright and dark regions indicates the presence of oscillations. Moreover, diagonal radiance enhancements (marked by dotted yellow lines and dashed blue lines in Figs. 2 and 3) are a signature of propagating disturbances. Thus, from such maps, it is possible to estimate periods and projected propagation speeds. From the SUMER data on both dates we measure a propagation speed of $\sim 75 \mathrm{~km} \mathrm{~s}^{-1}$ and a periodicity of about $15 \mathrm{~min}$. The propagation fronts are much more visible on the 8th April data (see top panel of Fig. 2) but are also visible on the 15th April data. In the case of the EIS data, no definitive conclusions can be drawn from the 8th April data (see bottom panel of Fig. 2), while propagating fronts are clearly visible in the 15th April data (see bottom panel of Fig. 3). However, both the propagation speeds and the periodicity are higher than for the SUMER data, with values of $\sim 125 \mathrm{~km} \mathrm{~s}^{-1}$ and $30 \mathrm{~min}$, respectively (see the dashed blue lines in the bottom panel of Fig. 3). The observed propagation speeds are actually a lower limit as the field lines may form an angle with the plane-of-sky. However, these angles are very likely small and the speeds are most probably subsonic. For the propagation speeds, we estimate the uncertainty to be around $10 \%$. The ratio of the Fe XII to Ne VIII propagation speeds, about 1.7 , is close to the ratio of the sound speeds of about 1.5 (at the two formation temperatures of 0.6 and $1.3 \mathrm{MK}$ ). The observed radiance perturbations have an amplitude of a few percent. No evidence of propagating disturbances can be found in the LOS velocity xt maps (not shown).

To better study the properties of the propagating disturbances seen in the enhanced-radiance xt slices in Figs. 2 and 3, we make use of wavelet analysis and focus on individual locations (heights) in the off-limb corona. For this purpose we extracted radiance vs. time curves at a given height by averaging over $9^{\prime \prime}$ in the $Y$ direction for both SUMER and EIS original (without filtering or contrast enhancement) radiance maps. In Fig. 4 (8th April) and Fig. 5 (15th April), we show representative examples of the type of oscillation measured at $Y=-995^{\prime \prime}\left(\sim 28^{\prime \prime}\right.$ above the limb). The top panels of Figs. 4 and 5 show the variation of the radiance (hereafter we will use the term radiance for trend-subtracted line radiance). Details on the wavelet analysis, which provides information on the temporal variation of a signal, are described in Torrence \& Compo (1998). For the convolution with the time series in the wavelet transform, we chose 
the Morlet function, as defined in Torrence \& Compo (1998). The light curves shown in the upper panels had their background trend removed by subtracting from the original time series a 60 -point $(\approx 18.1 \mathrm{~min}$ ) running average for 8th April data and a 100 -point $(\approx 30.2 \mathrm{~min})$ for SUMER and 150 -point $(\approx 45.3 \mathrm{~min})$ for the EIS running average for 15th April data. In the wavelet spectrum (middle-left panels), the cross-hatched regions are locations where estimates of oscillation periods become unreliable. This is the so-called cone-of-influence (COI, see Torrence \& Compo 1998). As a result of the COI, the maximum measurable period is shown by a dotted line in the global spectrum plots (middle-right panels). Above the global wavelet spectrum of Figs. 4 and 5 we show the prevalent period, measured at the location of the maximum of the global wavelet spectrum, together with an estimate of the probability that this oscillation is not due to noise. The probability estimate was calculated using the randomisation method with 200 permutations as outlined in detail in O'Shea et al. (2001).

Below the wavelet power spectrum, in the lower panels, we show the variation of the probability estimate, calculated using the randomisation technique, associated with the maximum power at each time in the wavelet power spectrum. The location of the maximum power is indicated by the over-plotted white lines in the middle-left panels. Figure 4 shows that the oscillations detected in the 8th April data have periodicities with maximum power at $\sim 14.1 \mathrm{~min}$ in Ne VIII (SUMER) and $\sim 13.9 \mathrm{~min}$ in Fe XII (EIS). Note that the wavelet technique reveals highly significant power in Fe XII (also at other heights), although no clear oscillation could be discerned by eye in the EIS enhanced radiance map (see bottom panel of Fig. 2). On the other hand, for 15th April data, which have a longer time series, Ne VIII shows its maximum power around $18 \mathrm{~min}$ and Fe XII shows maximum power around $30 \mathrm{~min}$, consistent with the results from the analysis of the xt slices. Also note that there are multiple peaks seen in the global wavelet spectra (right panels), which implies that the signal could be composed of multiple modes of oscillation. To study the oscillation behaviour at different heights we also look at the wavelet results at $Y=-1003^{\prime \prime}$ and plot the results in Figs. 6 and 7, respectively (available online only). For the 8th April data at $Y=-1003^{\prime \prime}$, SUMER reveals a strong peak at $14.2 \mathrm{~min}$, while EIS shows a peak at $15.15 \mathrm{~min}$. For the 15th April data at $Y=-1003^{\prime \prime}$ SUMER shows a strong peak at $20 \mathrm{~min}$, whereas EIS shows a peak at $30 \mathrm{~min}$. Finally, we also attempted to search for oscillations in the LOS velocity (by fitting the profiles obtained using the same binning as for the radiance wavelet analysis). No evidence of oscillations with an amplitude larger than $2 \mathrm{~km} \mathrm{~s}^{-1}$ can be found in the LOS velocity data. This is the accuracy achievable with SUMER when observing the Ne VIII line in the first order of diffraction (at the Signal to Noise values of relevance here).

\section{Conclusions}

The first observational detection of longitudinal waves came from analysing polarised brightness (density) fluctuations in white light data. Fluctuations with periods of about 9 min were detected in coronal holes at a height of about $1.9 R_{\odot}$ by Ofman et al. (1997) using the white light channel of UVCS/SoHO. In a follow-up study, Ofman et al. (2000b) determined the fluctuation periods to be in the range of 7 to $10 \mathrm{~min}$. The propagation speeds of the fluctuations indicated values in the range of 160 to $260 \mathrm{~km} \mathrm{~s}^{-1}$ at $2 R_{\odot}$, which is slightly slower than the acoustic speed at those heights. Deforest \& Gurman (1998), using
EIT $171 \AA$ reported detection of outwardly propagating radiance perturbations at distances of 1.01 to $1.2 R_{\odot}$, gathered in quasiperiodic groups of 3 to 10 periods, with periods of about 10 to $15 \mathrm{~min}$. The projected speeds are about 75 to $150 \mathrm{~km} \mathrm{~s}^{-1}$ and the relative amplitude (in density) was about 2 to $4 \%$. Usually these waves are observed propagating along the assumed coronal magnetic structures and, thus, along the magnetic field. Their speeds are usually much slower than the expected coronal Alfvén speed, which leads to their interpretation as longitudinally propagating slow magneto-acoustic waves. Slow magneto-acoustic waves follow magnetic field lines and propagate at the local sound speed. We detect the presence of long period oscillations with periods of 10 to $30 \mathrm{~min}$ in polar coronal holes within the range of 1 to $1.2 R_{\odot}$, with a clear signature of propagation with velocities from 75 to $125 \mathrm{~km} \mathrm{~s}^{-1}$, depending on the temperature of line formation. The measured propagation speeds are subsonic, indicating that they are slow magneto-acoustic in nature, which is consistent with earlier reports. Note that this detection has been confirmed through the analysis of data from two separate spectrometers on-board two different satellites. Thus we feel that this simultaneous detection makes the result very robust. We also find that the propagation speed in Fe XII $\left(\sim 125 \mathrm{~km} \mathrm{~s}^{-1}\right)$ is higher than that in Ne VIII $\left(\sim 75 \mathrm{~km} \mathrm{~s}^{-1}\right)$, as shown in the bottom panel of Fig. 3. This may be a temperature effect, as the ratio of the Fe XII to Ne VIII propagation speeds, about 1.7, is close to the ratio of the sound speeds. Different propagating speeds observed in different lines may also be interpreted as an indication of the presence of structures with different temperatures along the line of sight (e.g., weak plumes). The observed region can be either a bundle of magnetic threads of different temperatures, or have a transverse temperature profile. However, with the available data this statement remains a conjecture only. Finally, we note that the observed waves have no detectable signature in the LOS velocity, enforcing the idea of compressive longitudinal magneto-acoustic waves.

Acknowledgements. This work was supported by the Indo-German DST-DAAD joint project $\mathrm{D} / 07 / 03045$. The SUMER project is financially supported by DLR, CNES, NASA, and the ESA PRODEX programme (Swiss contribution). Hinode is a Japanese mission developed and launched by ISAS/JAXA, with NAOJ as domestic partner and NASA and STFC (UK) as international partners. It is operated by these agencies in co-operation with ESA and NSC (Norway). This work was partially supported by the WCU grant No. R31-10016 from the Korean Ministry of Education, Science and Technology. We thank the anonymous referee for useful comments and suggestions.

\section{References}

Banerjee, D., O'Shea, E., \& Doyle, J. G. 2000, Sol. Phys., 196, 63 Banerjee, D., O'Shea, E., Doyle, J. G., \& Goossens, M. 2001a, A\&A, 377, 691 Banerjee, D., O’Shea, E., Doyle, J. G., \& Goossens, M. 2001b, A\&A, 380, L39 Culhane, J. L., Harra, L. K., James, A. M., et al. 2007, Sol. Phys., 243, 19 Deforest, C. E., \& Gurman, J. B. 1998, ApJ, 501, L217

Kosugi, T., Matsuzaki, K., Sakao, T., et al. 2007, Sol. Phys., 243, 3

Ofman, L., Romoli, M., Poletto, G., Noci, G., \& Kohl, J. L. 1997, ApJ, 491, L111

Ofman, L., Nakariakov, V. M., \& Deforest, C. E. 1999, ApJ, 514, 441

Ofman, L., Nakariakov, V. M., \& Sehgal, N. 2000a, ApJ, 533, 1071

Ofman, L., Romoli, M., Poletto, G., Noci, G., \& Kohl, J. L. 2000b, ApJ, 529, 592

O’Shea, E., Banerjee, D., Doyle, J. G., Fleck, B., \& Murtagh, F. 2001, A\&A, 368, 1095

O’Shea, E., Banerjee, D., \& Doyle, J. G. 2006, A\&A, 452, 1059

O'Shea, E., Banerjee, D., \& Doyle, J. G. 2007, A\&A, 463, 713

Popescu, M. D., Banerjee, D., O’Shea, E., Doyle, J. G., \& Xia, L. D. 2005, A\&A, 442, 1087

Torrence, C., \& Compo, G. P. 1998, Bull. Amer. Meteo. Soc., 79, 61 Wilhelm, K., Curdt, W., Marsch, E., et al. 1995, Sol. Phys., 162, 189 
D. Banerjee et al.: Propagating waves in the corona, Online Material $p 1$

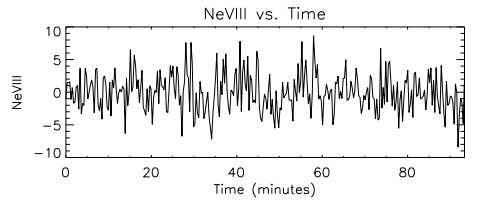

Global Period at max.

power $(<34.0 \mathrm{mi}$

Prob. level: $98.0 \%$
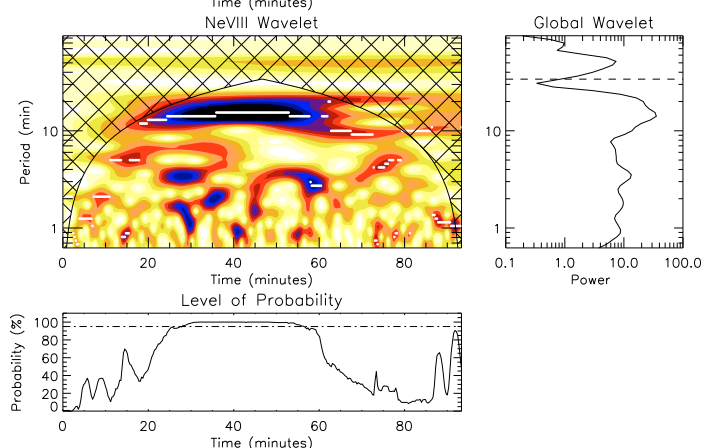

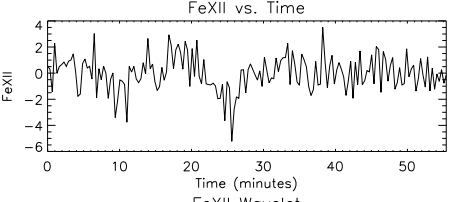

Global Period at ma

$=15.15 \mathrm{~min}$.

Prob. level: $99-100 \%$

年
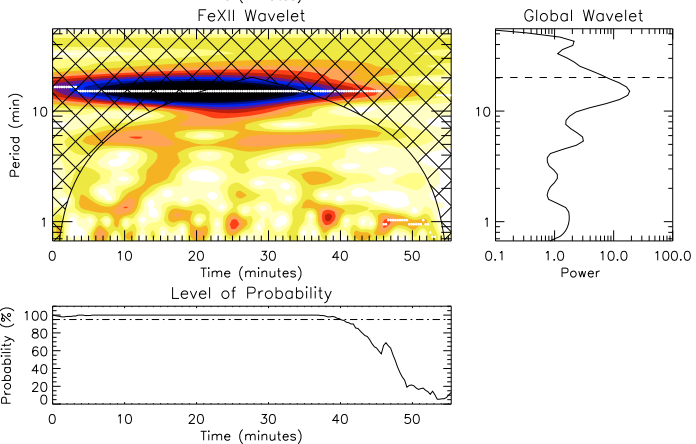

Fig. 6. Wavelet analysis for the 8th April data at Solar $Y \sim-1003^{\prime \prime}$ for both Ne VIII $770 \AA$ (left) and Fe XII $195 \AA$ (right). Both light curves are obtained by averaging over 9" along the slit. Panels as in Fig. 4.

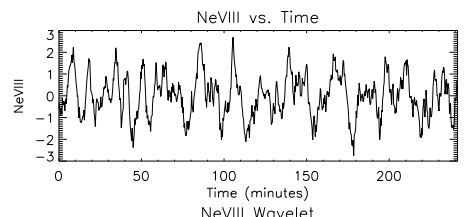

Global Period at max

power $(<88.0 \mathrm{~min}$.)

Prob. level: $99-100 \%$
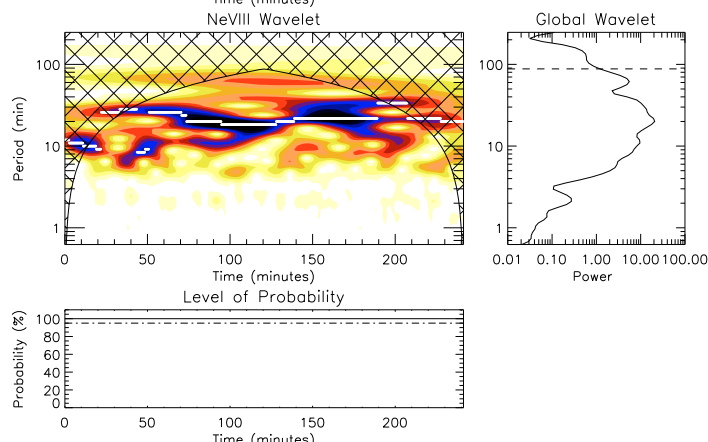

Global Period at max

power $(<85.7 \mathrm{~min}$.)

Prob. level: $99-100 \%$

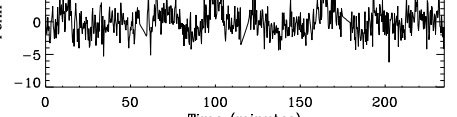
FeXII Wavelet
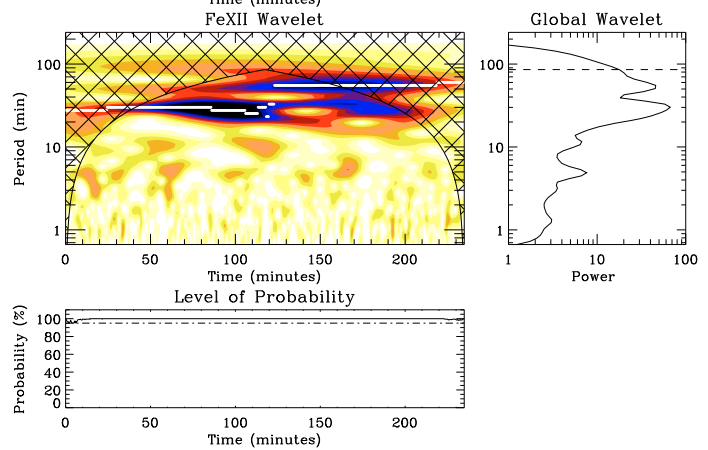

Fig. 7. Wavelet analysis for the 15th April data at Solar $Y \sim-1003^{\prime \prime}$ for both Ne VIII $770 \AA$ A (left) and Fe XII $195 \AA$ A (right). Both light curves are obtained by averaging over 9" along the slit. Panels as in Fig. 4. 